\title{
Phylogenetic analysis and development of molecular markers for five medicinal Alpinia species based on complete plastome sequences
}

Heyu Yang ${ }^{1,2+}$, Liqiang Wang ${ }^{2,3+}$, Haimei Chen ${ }^{2}$, Mei Jiang ${ }^{2}$, Wuwei Wu ${ }^{4}$, Shengyu Liu ${ }^{5}$, Jiehua Wang ${ }^{1 *}$ and Chang Liu $^{2^{*}}$

\begin{abstract}
Background: Alpinia species are widely used as medicinal herbs. To understand the taxonomic classification and plastome evolution of the medicinal Alpinia species and correctly identify medicinal products derived from Alpinia species, we systematically analyzed the plastome sequences from five Alpinia species. Four of the Alpinia species: Alpinia galanga (L.) Willd., Alpinia hainanensis K.Schum., Alpinia officinarum Hance, and Alpinia oxyphylla Miq., are listed in the Chinese pharmacopeia. The other one, Alpinia nigra (Gaertn.) Burtt, is well known for its medicinal values.

Results: The four Alpinia species: A. galanga, A. nigra, A. officinarum, and A. oxyphylla, were sequenced using the Nextgeneration sequencing technology. The plastomes were assembled using Novoplasty and annotated using CPGAVAS2. The sizes of the four plastomes range from 160,590 bp for A. galanga to 164,294 bp for A. nigra, and display a conserved quadripartite structure. Each of the plastomes encodes a total of 111 unique genes, including 79 protein-coding, 28 tRNA, and four rRNA genes. In addition, 293-296 SSRs were detected in the four plastomes, of which the majority are mononucleotides Adenine/Thymine and are found in the noncoding regions. The long repeat analysis shows all types of repeats are contained in the plastomes, of which palindromic repeats occur most frequently. The comparative genomic analyses revealed that the pair of the inverted repeats were less divergent than the single-copy region. Analysis of sequence divergence on protein-coding genes showed that two genes ( $a c c D$ and ycf1) had undergone positive selection. Phylogenetic analysis based on coding sequence of 77 shared plastome genes resolves the molecular phylogeny of 20 species from Zingiberaceae. In particular, molecular phylogeny of four sequenced Alpinia species (A. galanga, A. nigra, A. officinarum, and A. oxyphylla) based on the plastome and nuclear sequences showed congruency. Furthermore, a comparison of the four newly sequenced Alpinia plastomes and one previously reported Alpinia plastomes (accession number: NC_048461) reveals 59 highly divergent intergenic spacer regions. We developed and validated two molecular markers Alpp and Alpr, based on two regions: petN-psbM and psaJ-rp/33, respectively. The discrimination
\end{abstract}

\footnotetext{
* Correspondence: jiehuawang@tju.edu.cn; cliu6688@yahoo.com

${ }^{\dagger}$ Heyu Yang and Liqiang Wang contributed equally to this work.

'School of Environmental Science and Engineering, Tianjin University, 300072

Tianjin, China

${ }^{2}$ Institute of Medicinal Plant Development, Chinese Academy of Medical

Sciences and Peking Union Medical College, 100193 Beijing, People's

Republic of China

Full list of author information is available at the end of the article
}

(C) The Author(s). 2021 Open Access This article is licensed under a Creative Commons Attribution 4.0 International License, which permits use, sharing, adaptation, distribution and reproduction in any medium or format, as long as you give appropriate credit to the original author(s) and the source, provide a link to the Creative Commons licence, and indicate if changes were made. The images or other third party material in this article are included in the article's Creative Commons licence, unless indicated otherwise in a credit line to the material. If material is not included in the article's Creative Commons licence and your intended use is not permitted by statutory regulation or exceeds the permitted use, you will need to obtain permission directly from the copyright holder. To view a copy of this licence, visit http://creativecommons.org/licenses/by/4.0/. The Creative Commons Public Domain Dedication waiver (http://creativecommons.org/publicdomain/zero/1.0/) applies to the data made available in this article, unless otherwise stated in a credit line to the data. 
success rate was $100 \%$ in validation experiments.

Conclusions: The results from this study will be invaluable for ensuring the effective and safe uses of Alpinia medicinal products and for the exploration of novel Alpinia species to improve human health.

Keywords: Plastome, Alpinia, Phylogenomic analysis, Species authentication

\section{Background}

Zingiberaceae is the largest plant family in the order Zingiberales [1]. It contains about 1,587 species and 52 genera (The Plant List; last accessed: February 2021). The family provides essential natural resources to humans, including many useful products for food, spices, medicines, dyes, perfume, and aesthetics [2, 3]. Alpinia Roxb. is the largest, most widely distributed, and most taxonomically complex genus in the Zingiberaceae, including 230 species occurring throughout tropical and subtropical Asia [4]. Alpinia comprises approximately 54 species in China. Many of the Alpinia species are wellknown medicinal herbs. Other Alpinia species have been widely used for bioprospection of plant essential oils for medicinal uses [5].

The Chinese pharmacopeia (2020 version) contains 15 Zingiberaceae species belonging to five genera, and four of the 15 species belong to the genus Alpinia. These four species are A. galanga, A. officinarum, A. oxyphylla, and $A$. hainanensis. The first species, A. galanga, also called "Hong Dou Kou," has been used to manage dyspepsia, fever, urinary incontinence, halitosis, and hoarseness of voice in throat infections [6]. The second species, $A$. officinarum, has been used to relieve stomachache, treat colds, invigorate the circulatory system, and reduce swelling. Many chemical constituents have been isolated from this plant, including monoterpenes, diarylheptanoids, flavonoids, phenylpropanoids, and neolignans [7]. The third species, A. oxyphylla, also called "Yi Zhi," is widely used to treat dyspepsia, diarrhea, abdominal pain, spermatorrhea, kidney asthenia, and poor memory [8]. The fourth species, A. hainanensis is native to the Hainan Island in Southern China. It has been used for its anti-emetic and stomachic mechanism of action [9]. Another species, A. nigra has been used traditionally to treat bronchitis, gastric ulcers, parasitic intestinal infections. However, it is not included in the Chinese pharmacopeia (2020 version) [10]. The morphological identification of these species is problematic. Misidentification will undermine the efficacy and safety of medicinal products developed from them [11, 12]. Additionally, the genetic divergence among these species and the complex evolutionary history of the genus are often poorly understood, making it difficult for the bioprospecting of medicinal Alpinia species.

Plastomes provide a robust framework that can be used to examine phylogenetic relationships among plants and provide new probes for species identification $[13,14]$. Their comparatively conserved and well-defined genome structures allow the investigation of a wide range of crucial issues. Initially, genetic studies focused on understanding each plastid genome, particularly of the overview features, such as genome size, gene content, and sequence repetition [15]. Lately, the crucial role of the plastomes in the evolution and impact for speciation has become obvious demonstrated by the sequence divergence, large inversion, differences in coding and intergenic regions, and evolutionary analysis $[16,17]$.

To date, complete plastomes are available from more than 100 Zingiberaceae species, including four Alpinia species. Recently, a complete plastome of $A$. oxyphylla (NC_035895) was analyzed, and the plastome shared the highest sequence similarity of $>90 \%$ to that of A. zerumbet [18]. Based on the single nucleotide polymorphism (SNP) matrix among 28 whole plastomes, including a plastome (NC_048461) of A. hainanensis and two plastomes (NC_035895, MK262729) of A. oxyphylla, a phylogenetic analysis showed that Alpinia and Amomum are closely related in the family Zingiberaceae [19]. Such results provided useful information to understand the Alpinia evolution. However, they have not focused on the species that are widely used for their medicinal values and there is no phylogenetic analysis using nuclear markers in Alpinia species.

In previous reports, phylogeny, biodiversity assessment within populations, and the authentication of Alpinia species have been studied using several molecular markers. Nuclear ribosomal DNA internal transcribed spacers (ITS) sequences have been used as markers to distinguish A. galanga from its adulterants (Zhao et al. 2001). Efficacy of DNA barcode internal transcribed spacer 2 (ITS2) was tested on species identification of Alpinia species from Peninsular Malaysia [20]. Also, the information of genetic relatedness was developed using seven plastid barcoding loci among wild Alpinia nigra (Gaertn.) B.L. Burtt populations [21].

Lately, chloroplast-derived DNA markers were developed to authenticate medicinal plants. One example is SNPs and insertion-deletion mutations (Indels) of the intergenic regions in the plastome of Panax ginseng species [22, 23]. However, there are no systematic studies to develop molecular markers for medicinal Alpinia 
species. Our short-term goal is to understand the taxonomic relationship of medicinal Alpinia species and develop molecular markers for their discrimination. And our long-term goal is to develop a method for ensuring the efficacy and safety of Alpinia medicinal products and identify new Alpinia species for medicinal uses. In this study, we reported and compared the four complete plastome sequences of $A$. galanga, A. nigra, A. officinarum, and $A$. oxyphylla sampled from Guangxi, China. The phylogenetic relationships of medicinal Alpinia species were studied based on plastome sequences and single-copy nuclear genes. Molecular markers based on plastomes were furtherly developed for the discrimination of the five Alpinia species and were validated successfully.

\section{Results}

\section{Features of the Alpinia species plastomes}

The plastomes are circular structures of $160,590 \mathrm{bp}$ ( $A$. galanga), 164,294 bp (A. nigra), 162,140 bp (A. officinarum), and 161,394 bp (A. oxyphylla) long. The schematic representation of the plastomes is shown in Fig. 1 and Figures S1, S2 and S3, respectively. The four plastomes display the typical quadripartite characters and show a high degree of conservation in organization and structure. They consist of a Large Single-Copy (LSC) region $(87,267-88,970 \mathrm{bp})$ and a Small Single-Copy (SSC) region $(15,349-17,908 \mathrm{bp})$, which were separated by two Inverted Repeat (IR) regions (27,490-29,951 bp) (Table 1). The overall GC contents of A. galanga, A. nigra, $A$. officinarum, and $A$. oxyphylla plastomes are $36.24 \%, 35.98 \%, 36.14 \%$, and $36.16 \%$, respectively. Whereas the GC contents of their coding sequences (CDS) regions are $37.13 \%, 36.91 \%, 36.88 \%$, and $36.95 \%$, respectively (Table $\mathrm{S} 1$ ), and are somewhat higher than those of the whole plastomes.

All of the four Alpinia plastomes encode a set of 111 unique genes with identical gene order and gene clusters. Seventy-nine of these are protein-coding genes, 28 are tRNA genes, and four are rRNA genes (Tables S2, S3, S4 and S5). Fourteen genes $(a t p \mathrm{~F}, n d h \mathrm{~A}, n d h \mathrm{~B}, p e t \mathrm{~B}$, pet $\mathrm{D}$, $r p l 2$, rpoC1, rps16, trnA-UGC, trnC-ACA, trnE-UUC, $\operatorname{trnK}$-UUU, trnL-UAA, trnS-CGA) contain one intron, while three genes, $c l p \mathrm{P}, y c f 3$ and $r p s 12$, possess two introns (Tables S6, S7, S8 and S9, Figures S4, S5, S6 and S7). In particular, the rps 12 is generated by trans-splicing and has three exons (Figures S4, S5, S6 and S7, lower panels). We also detected 2-262 heteroplasmic sites with minor allele frequency (MAF) of 0.6-1\% in four sequenced Alpinia species (Figures S8, S9, S10 and S11).

\section{Sequence repetition in the Alpinia plastomes}

Comparative analysis of sequence repetition between all four plastomes found that the overall distribution, types, and numbers of repeats are highly similar among the plastomes. Simple sequence repeats (SSRs) are sequences composed of repeats with motifs from 1 to $6 \mathrm{bp}$ in length. They are widespread in plastomes and widely utilized for species identification, genetic linkage construction, and molecular breeding [24]. A total of 293-296 SSRs were found in the Alpinia plastomes (Table 2). The most abundant mononucleotide SSRs are polyadenine or polythymine repeat types. Interestingly, hexanucleotide SSRs were not found in the plastomes of $A$. galanga and A. oxyphylla but were detected in the other two Alpinia plastomes. Further analysis of the size and location of the different SSR units and comparison revealed that the composite SSR was variable among the four species, while the dinucleotide repeat of AT was conserved (Tables S10, S11, S12, S13 and S14).

Long repeat analyses of four sequenced plastomes showed that 45-49 dispersed repeats were detected, which belong to forward, reverse, complementary and palindromic repeats (Table 3). Forward (direct) and palindrome (inverted) repeats were considerably higher in number than reverse and complement repeats. The majority of these repeats with the repeat length range from 30 to 49 bp were located in intergenic spacer (IGS) regions (Tables S15, S16, S17 and S18). We found the dispersed repeats within those genes were mostly located in the exons but not in the introns. They can potentially facilitate structural rearrangements and develop variability among plastomes in a population [25].

On average, the numbers of detected tandem repeats range from 28 in A. officinarum up to 33 in A. oxyphylla. The copy numbers of these repeats range from 1.9 to 5.3 copies per tandem repeat, and the repeat sizes range from 30 to 158 bp per copy (Tables S19, S20, S21 and S22). The tandem repeats were found extensively in the IGS regions.

\section{Expansion of the IR regions in Alpinia plastomes}

The variations in the single-copy and IR regions' sizes and boundaries commonly cause evolutionary events such as contraction and expansion in the plastome architecture [26]. We compared the IR and single-copy region boundaries among six species, including one Zingiber species and the five Alpinia plastomes, the four Alpinia sequenced in our research, and A. hainanensis. Two A. oxyphylla genomes sequences previously were included in the analysis. Some divergences were identified among the plastomes of four Alpinia species and Z. spectabile (Fig. 2). Particularly, IR expansions were found in the LSC/IRa boundary of the four Alpinia species, which included the complete rps 19 gene in the IRs of these species.

In contrast, the rps 19 gene is located in the LSC region of $Z$. spectabile. The distances between the border of rps 19 and the IR/LSC junction were 13, 160, 119, 129, and 129 


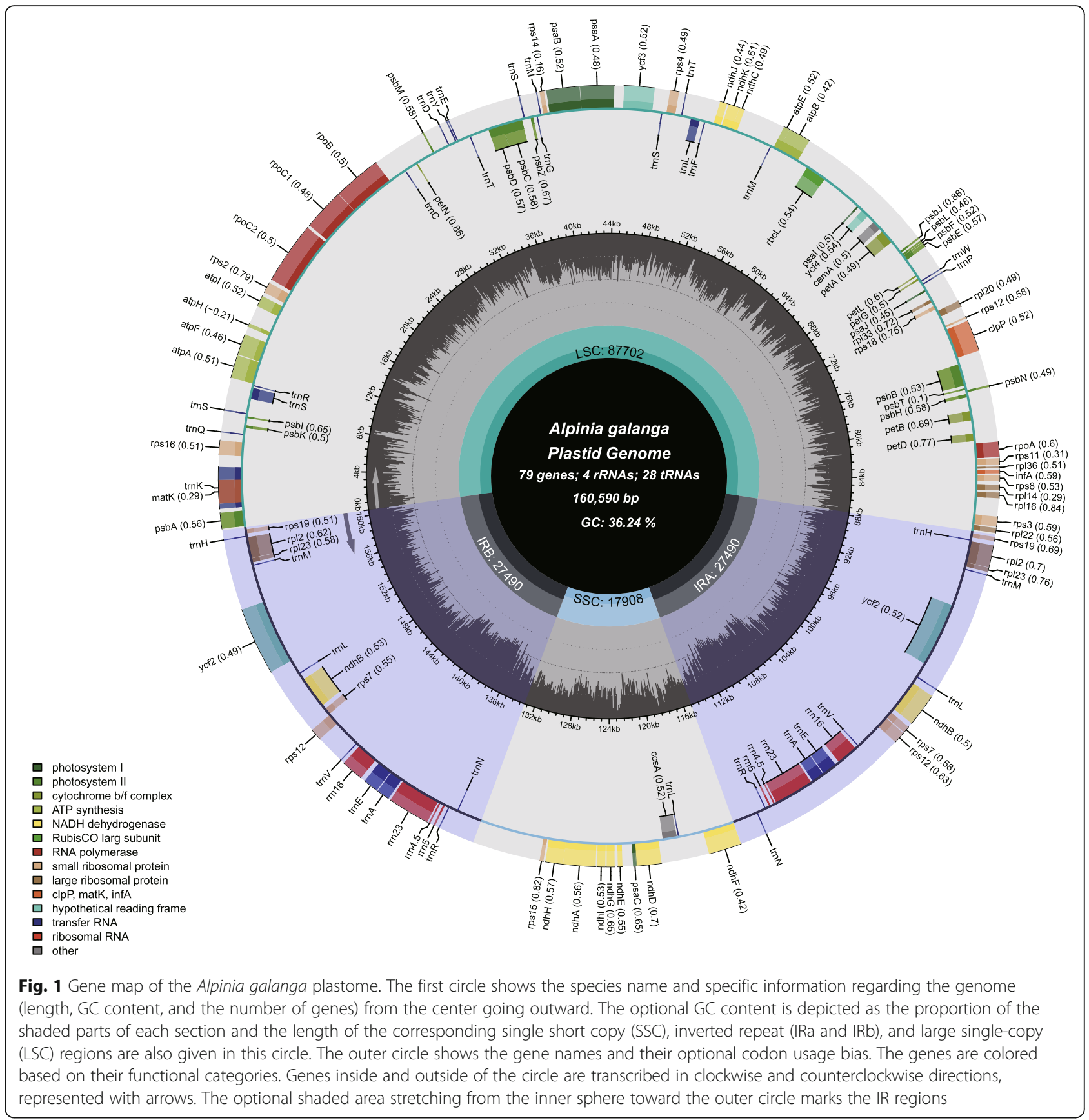

bps in the plastomes of $Z$. spectabile, A. galanga, A. nigra, A. officinarum, and A. oxyphylla, respectively. Another interesting observation is that the $y c f 1$ gene is localized in the $\mathrm{IRb}$ region. The $y c f 1$ gene sequence is significantly longer in the Alpinia species, 3944 bp for A. nigra, 1428 bp for $A$. galanga, and 3944 bp for $A$. nigra, compared with that of $Z$. spectabile (924 bp) (Fig. 2).

\section{Hypervariable regions}

We compared the plastome sequences of five Alpinia species, among them, A. oxyphylla with three accessions, and Zingiber species to determine the overall variations among the Alpinia and Zingiber species. As shown in Fig. 3, the plastomes are highly conserved among these species. The IR regions were less divergent than the LSC and SSC regions. The coding regions were more conserved than the noncoding regions. However, ndhA, pet $\mathrm{B}, y c f 1$, and $y c f 2$ genes showed a relatively high degree of sequence divergence. In contrast, the IGS regions were highly diverse, particularly in the following regions: rps16-trnQ, pet $\mathrm{N}-p s b \mathrm{M}, p s a \mathrm{C}-n d h \mathrm{E}, a c c \mathrm{D}-p s a \mathrm{I}, p s a \mathrm{~J}-$ rpl33, matK-rps16, psbH-petB (Fig. 3). 
Table 1 General features of the four Alpinia plastomes

\begin{tabular}{lllll}
\hline & A. galanga & A. nigra & A. officinarum & A. oxyphylla \\
\hline GeneBank Accession number & MK940825 & MK940826 & MK940823 & MK940824 \\
Plastome Length (bp) & 160,590 & 164,294 & 162,140 & 161,394 \\
LSC $^{\text {a }}$ Length (bp) & 87,702 & 88,970 & 87,267 & 87,293 \\
SSC ${ }^{\text {b }}$ Length (bp) & 17,908 & 15,422 & 15,349 & 16,177 \\
IR $^{c}$ Length (bp) & 27,490 & 29,951 & 29,762 & 28,962 \\
Number of Genes & 135 & 135 & 135 & 135 \\
\hline
\end{tabular}

aSC Large Single-Copy region

${ }^{\mathrm{b}}$ SSC Small Single-Copy region

CIR Inverted Repeat region

Table 2 Type and number of Simple-Sequence Repeat (SSRs) found in the four Alpinia plastomes

\begin{tabular}{|c|c|c|c|c|c|}
\hline \multirow[t]{2}{*}{ Type } & \multirow[t]{2}{*}{ Repeat Unit } & \multicolumn{4}{|c|}{ Numbers of Repeats } \\
\hline & & A. galanga & A. nigra & A. officinarum & A. oxyphylla \\
\hline \multirow[t]{2}{*}{ Mono- } & $\mathrm{A} / \mathrm{T}$ & 172 & 179 & 178 & 177 \\
\hline & $C / G$ & 7 & 6 & 7 & 7 \\
\hline \multirow[t]{3}{*}{ Di- } & AT/AT & 62 & 65 & 64 & 64 \\
\hline & $\mathrm{AC/GT}$ & 2 & 1 & 1 & 1 \\
\hline & $\mathrm{AG} / \mathrm{CT}$ & 21 & 21 & 22 & 0 \\
\hline \multirow[t]{5}{*}{ Tri- } & AAT/ATT & 1 & 1 & 0 & 0 \\
\hline & $\mathrm{AAG} / \mathrm{CT}$ & 3 & 3 & 3 & 3 \\
\hline & AG C/CTG & 0 & 0 & 0 & 0 \\
\hline & $\mathrm{AG} \mathrm{G} / \mathrm{CCT}$ & 1 & 0 & 1 & 1 \\
\hline & $\mathrm{ACT} / \mathrm{AGT}$ & 1 & 1 & 0 & 0 \\
\hline \multirow[t]{7}{*}{ Tetra- } & AAAC/GTT & 1 & 0 & 1 & 1 \\
\hline & AAAG/CTTT & 3 & 4 & 1 & 3 \\
\hline & AAAT/ATTा & 10 & 6 & 9 & 9 \\
\hline & AACT/AGTT & 1 & 1 & 1 & 1 \\
\hline & AATG/ATTC & 1 & 2 & 2 & 1 \\
\hline & AATT/AATT & 3 & 1 & 1 & 1 \\
\hline & ACAT/ATGT & 1 & 1 & 1 & 1 \\
\hline \multirow[t]{5}{*}{ Penta- } & AAAAT/ATTाT & 0 & 0 & 0 & 0 \\
\hline & AAATC/ATTTG & 0 & 0 & 0 & 0 \\
\hline & AAATT/AATTT & 0 & 0 & 1 & 1 \\
\hline & AACCC/GGGTT & 0 & 0 & 0 & 0 \\
\hline & AATAT/ATATT & 3 & 1 & 0 & 0 \\
\hline \multirow[t]{6}{*}{ Hexa- } & AATATT/AATATT & 0 & 0 & 0 & 0 \\
\hline & AAATAT/ATATTT & 0 & 0 & 0 & 0 \\
\hline & AAGAGG/CCTCTT & 0 & 0 & 0 & 0 \\
\hline & ACTATC/AGTGAT & 0 & 0 & 0 & 0 \\
\hline & AAATTT/AAATTT & 0 & 0 & 1 & 0 \\
\hline & AAAATT/AATTTT & 0 & 1 & 0 & 0 \\
\hline Total No. & -- & 293 & 294 & 296 & 293 \\
\hline
\end{tabular}


Table 3 Dispersed repeat sequences identified in the four Alpinia plastomes. REPuter was used to recognize repeat sequences with length $\geq 30 \mathrm{bp}$ and identity $\geq 90 \%$. F forward, $P$ palindromic, $R$ reverse, and $C$ complement

\begin{tabular}{|c|c|c|c|c|c|}
\hline Type & Size (bp) & A. galanga & A. nigra & A. officinarum & A. oxyphylla \\
\hline \multirow[t]{5}{*}{$\mathrm{F}$} & $30-39$ & 2 & 5 & 4 & 5 \\
\hline & $40-49$ & 12 & 11 & 7 & 7 \\
\hline & $50-59$ & 1 & 1 & 2 & 2 \\
\hline & $60-69$ & 0 & 0 & 2 & 2 \\
\hline & $\geq 70$ & 3 & 5 & 0 & 0 \\
\hline \multirow[t]{5}{*}{ P } & $30-39$ & 8 & 3 & 18 & 14 \\
\hline & $40-49$ & 13 & 14 & 8 & 8 \\
\hline & $50-59$ & 1 & 1 & 3 & 3 \\
\hline & $60-69$ & 0 & 0 & 2 & 2 \\
\hline & $\geq 70$ & 4 & 3 & 0 & 0 \\
\hline \multirow[t]{5}{*}{$R$} & $30-39$ & 1 & 1 & 0 & 4 \\
\hline & $40-49$ & 0 & 1 & 0 & 0 \\
\hline & $50-59$ & 0 & 0 & 0 & 0 \\
\hline & $60-69$ & 0 & 0 & 0 & 0 \\
\hline & $\geq 70$ & 0 & 0 & 0 & 0 \\
\hline \multirow[t]{5}{*}{ C } & $30-39$ & 0 & 0 & 2 & 2 \\
\hline & $40-49$ & 0 & 0 & 0 & 0 \\
\hline & $50-59$ & 0 & 0 & 0 & 0 \\
\hline & $60-69$ & 0 & 0 & 0 & 0 \\
\hline & $\geq 70$ & 0 & 0 & 0 & 0 \\
\hline Total & -- & 45 & 46 & 49 & 49 \\
\hline
\end{tabular}

Hypervariable regions can be used to resolve phylogenies and to discriminate closely related plant species [27]. The pairwise comparison of intergenic spacer regions was conducted to identify divergence hotspot regions among the five Alpinia species using the Kimura 2-parameter (K2p) model. The average K2p distance ranged from 0.00 to 6.793 among 59 IGSs extracted from these species. Among them, the IGS regions $p s b \mathrm{E}-$ pet $\mathrm{L}$, pet $\mathrm{N}-p s b \mathrm{M}, a c c \mathrm{D}-p s a \mathrm{I}$, pet $\mathrm{D}-r p o \mathrm{~A}$ showed the largest distances of $6.79,6.32,5.51$, and 5.27, respectively (Fig. 4, Table S23).

\section{Phylogenomic analyses based on plastome data}

The availability of more complete plastome sequences of Alpinia species allows us to conduct phylogenomic analyses with higher resolution in Zingiberaceae (Fig. 5). We performed a phylogenetic analysis using the Maximum likelihood (ML) method based on DNA sequences of 77 genes shared among 20 species from Zingiberaceae, including the four Alpinia species sequenced in the study (Table S24). The sister genus of Alpinia is Amomum with a Bootstrap score (BS) of 100. The species of Alpinia are distributed in two main clades. The first clade (BS: 100) is formed by $A$. galanga and A. nigra, both medicinal species. The second clade (BS: 100) contains most of the species sampled to date. These species are from the tropical and subtropical geographic regions and many species are of medicinal value. Two accessions of $A$. oxyphylla are clustered together (BS: 99), which are subsequently clustered together with $A$. officinarum (Fig. 5). Also, the phylogenetic positions of A. galanga and $A$. nigra were reported for the first time based on the plastomes. The bootstrap scores are high for all branches indicating the high degree of reliability of the phylogenetic tree.

\section{Phylogenetic analysis based on nuclear markers}

The low-coverage sequence data generated from this study allowed us to perform phylogenetic analysis using additional nuclear markers. We extracted nuclear genes from sequence data among the Angiosperms-mega 353 gene set [28]. Among these genes, 352, 353, 353, 352 genes had mapped reads, and the reads mapped to 173 , 28, 93, 59 genes were assembled into contigs for A. galanga, $A$. nigra, $A$. officinarum, and $A$. oxyphylla, respectively. Among these assembled contigs, only four genes (AT4G04780, AT3G53760, AT5G53800, AT1G06240) were shared among the four species. These four genes were used to construct a phylogenetic tree using the same method as that for the complete plastome sequences. The reconstructed ML tree with these four genes was well resolved overall. And two of the nodes were supported with bootstrap values of 75 and $69 \%$ (Fig. 6). Among the four Alpinia species, $A$. galanga was sister to $A$. nigra, and $A$. officinarum was sister to $A$. oxyphylla. To compare if the relationships in both the nuclear and plastome trees are consistent, the phylogenetic analysis of plastomes with the same taxon sampling as the nuclear tree was conducted. The relevant result was consistent with the results of phylogenetic inferences obtained with nuclear markers (Fig. 6). This approach enabled us to define further the phylogenetic relationship between the four Alpinia species using nuclear genes.

\section{Variation and evolutionary selection of protein-coding genes}

Purifying/positive selection analyses of 77 protein-coding genes in the Alpinia plastomes showed that most genes exhibited $\omega$ values less than 0.5 . Five genes $(p s b \mathrm{I}, \operatorname{pet} \mathrm{N}$, $p s b \mathrm{M}$, pet $\mathrm{L}$, and $p s b \mathrm{~T}$ ) had the lowest $\omega$ ratios close to 0 . In contrast, the $\omega$ values of $y c f 2, a c c \mathrm{D}, r p l 23, r p s 7$, and $y c f 1$ were more than 1.00 , respectively (Table S25). The results showed that the genes $a c c \mathrm{D}$ and $y c f 1$ were under positive selection. The likelihood ratio test identified three and five amino acid sites in $a c c \mathrm{D}$ and $y c f 1$ that were positively selected (under posterior probability > 


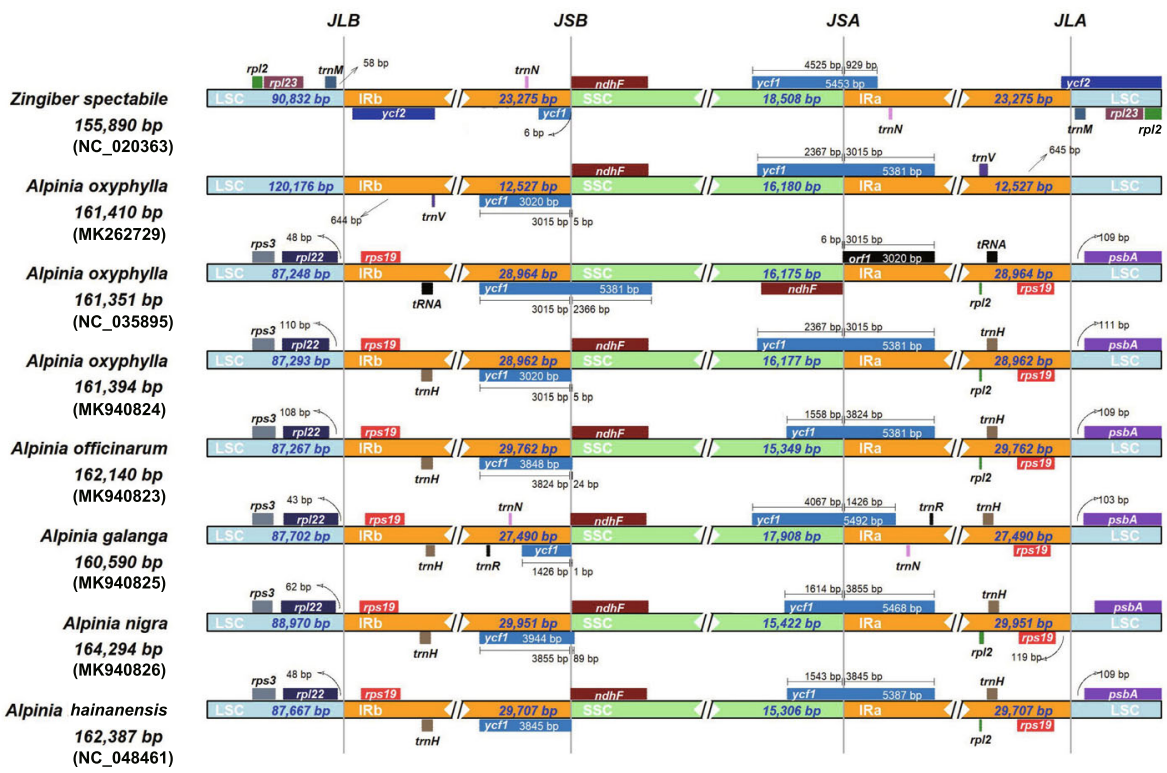

Fig. 2 Analysis of the Contraction and Expansion of the IR regions. Schematic representation of the boundary areas of LSC (light blue), IRa (yellow), SSC (green), and IRb (yellow) regions for Zingiber spectabile, A. galanga, A. hainanensis, A. nigra, A. officinarum, and three A. oxyphylla accessions. The species names are shown to the left. The long horizontal blocks represent the plastomes. The name and length of each region are shown. The junction sites between LSC, IRa, SSC, and IRb are marked with the perpendicular dashed line. The genes rp/22 (dark blue), rps 19 (light red), ycf1 (blue), ndhF (red), and psbA (light purple) are shown above the plastomes. The numbers above the gene features denote the distance between the gene borders, either the start or end of genes and the junction sites

0.95), respectively (Table 4). These sites are also highly polymorphic in the two genes.

\section{Molecular marker development based on Alpinia plastomes}

To discriminate the five medicinal Alpinia species, we selected two hypervariable IGS regions, pet $\mathrm{N}-p s b \mathrm{M}$, and psaJ-rpl33, to develop two DNA markers named Alpp and Alpr, respectively. The PCR primers used to amplify these two markers are shown in Table S26. PCR amplification of total DNAs from all five medicinal species samples resulted in products having expected size (Fig. 7, Figure S12, Table S27). The DNA fragments were extracted from each band and then subjected to Sanger sequencing. The sequencing results were identical to the expected sequences (Figures S13 and S14). Marker Alpp, derived from the pet N-psbM IGS region, has two specific SNP loci and one Indel loci. These three variable loci can be used to differentiate three of the five Alpinia species, except $A$. officinarum and $A$. oxyphylla. The marker Alpr, derived from the $p s a \mathrm{~J}$-rpl33 IGS region. It has two SNP loci and one Indel loci. When using the SNP and Indel loci from both Alpp and Alpr, all the five species can be differentiated successfully (Fig. 8). We also have tested the new primers on all ten available Alpinia plastomes obtained from NCBI and this study in silico. These markers can discriminate all eight species based on the SNP and Indel loci from both Alpp and Alpr (Figures S15 and S16).

\section{Discussion}

Here, we studied five medicinal Alpinia species, Alpinia galanga, A. hainanensis, A. officinarum, A. oxyphylla, and $A$. nigra. We sequenced the four plastomes of these five species. Three of them belonging to Alpinia galanga, $A$. officinarum, and $A$. nigra were reported for the first time. Two plastomes of $A$. oxyphylla were released during the study period. We carried out a detailed analysis of the genome features, performed the phylogenetic analysis with plastid proteomes and nuclear makers. Lastly, we developed a set of two primers that can distinguish these five medicinal species.

Compared to the plastomes of previously published Alpinia species, all the plastomes presented in this study exhibited consistent genomic structure, gene order, and content. And there are no significant structural rearrangements, such as inversions or gene relocations (Fig. 1, Table S1). The size of the A. oxyphylla plastomes (MK940824) in this study is almost identical to the other two reported plastomes, which were 161,394 bp (MK940824), 161,410 bp (MK262729) [19], and 161,351 bp (NC_035895) [18]. We found that the most abundant mononucleotide SSRs are of polyadenine or polythymine repeat types in the four Alpinia species, consistent with those reported previously [29]. Plastomes 


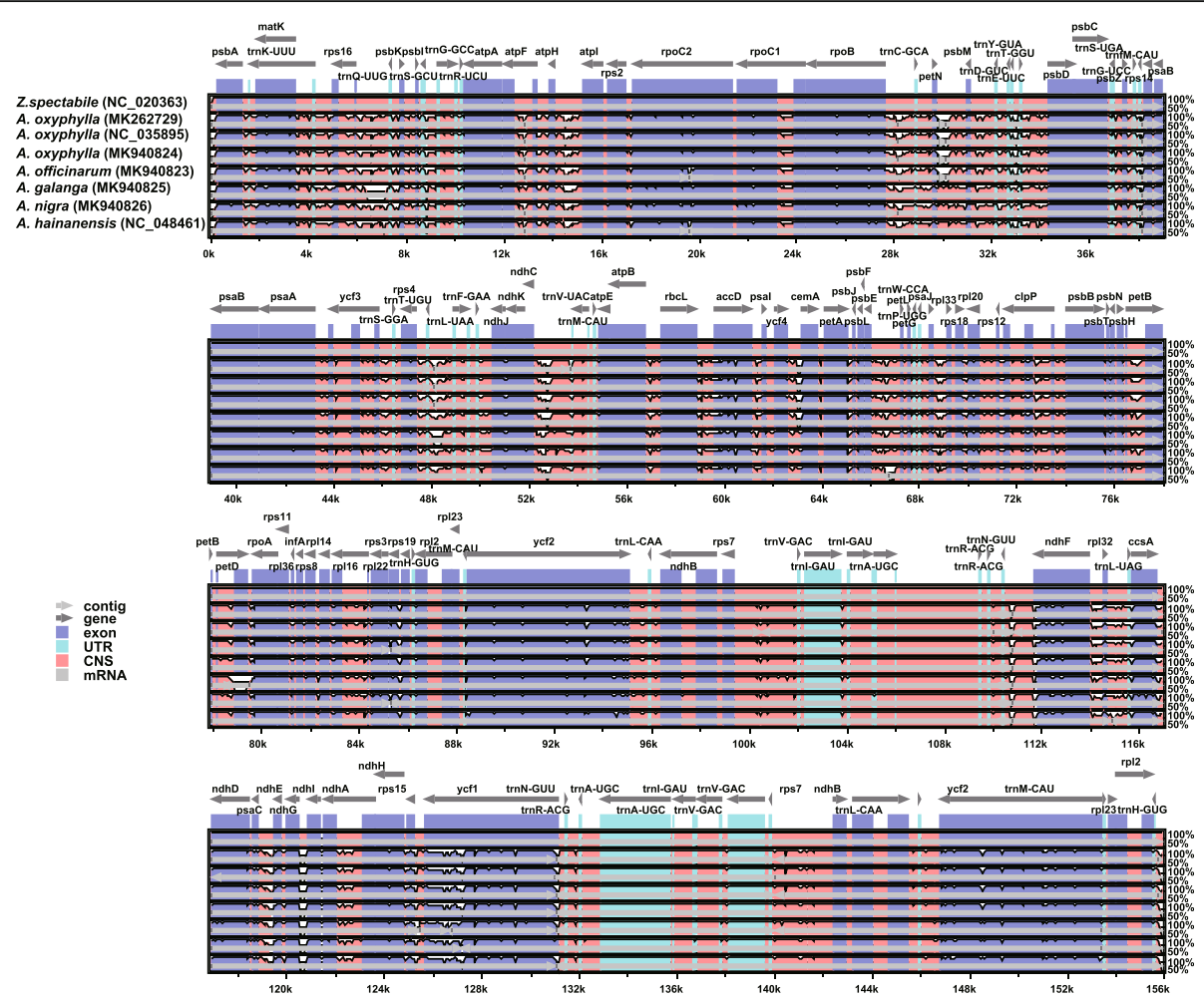

Fig. 3 Sequence identity plot of the seven Alpinia plastomes with Zingiber spectabile as a reference by mVISTA. The species names are shown to the left. The grey arrows above the alignment indicate the transcription direction of genes. In the alignment box, the blue color box indicates protein-coding, the pink color box shows the conserved noncoding sequence, and the light green box indicates tRNAs and rRNAs. The x-axis represents the positions in the cp. genome, and the Y-scale represents the percent identity ranging from 50-100\%

are well-arranged, except for the expansion of the IR regions in the Alpinia species. Judged from comparative analysis with the plastome of $Z$. spectabile as a reference, the IR lengths of all the four Alpinia species plastomes were all increased to $\geq 160 \mathrm{kbp}$. Also, one evidence supporting this expansion is that the rps 19 gene has moved to the IR regions. In other species of Alpinia, plastomes reported so far $[18,19]$, the entire rps 19 gene is also localized in the IR region, which is consistent with our findings. The analysis revealed that the four Alpinia species sequenced in our study have heteroplasmy sites in their plastomes. However, the positions of these detected heteroplasmic sites and two developed molecular markers did not overlap.

Classifications and phylogenetic analysis among Zingiberaceae were previously reported based on morphological features and DNA sequences of the nuclear internal transcribed spacer (ITS) and plastid matK regions [1, 30-32]. We use four new plastome sequences to define the position of four Alpinia species in Zingiberaceae. The new accession of $A$. oxyphylla sequenced (MK 940,824) in this study was most closely related to the other one $A$. oxyphylla plastomes reported previously (NC_035895) [19]. To date, the phylogenetic inference of Alpinia species has mainly relied on plastid markers $[18,19]$ and few multi-copy nuclear ribosomal regions such as ITS [33]. Our phylogenetic analysis results create reliable phylogenies of the four Alpinia species sequenced by us using the nuclear markers for the first time. In addition, phylogenetic analysis using plastome and nuclear sequences revealed the identical phylogenetic relationships for the four Alpinia species.

Because of the lack of mobility, plants must deal with the challenge of abiotic stresses, such as soil salinity, drought, and extreme temperature. Many genes from plastomes, such as $c l p \mathrm{P}$ [34], $r b c \mathrm{~L}$ [35], and mat K [36], $y c f 1$ and $y c f 2$ [37], have been positively selected. The positive selection of the plastome genes may serve as an adaptive evolution for adjusting to environmental changes. In the selective pressure analysis, five genes were positively selected, and their selection might reflect the adaptive evolution of these Alpinia species. The results are consistent with the reports that $a c c \mathrm{D}$ and $y c f 1$ evolved under positive selection in the Zingiber plastomes [37]. Particular amino acids were identified to have been positively selected in two genes, $a c c \mathrm{D}$, and $y c f 1$. For example, the plastid $a c c \mathrm{D}$ is an essential gene required for leaf development [38], and the $y c f 1$ is crucial for plant viability [39]. In the current research, all four Alpinia species studied distributed in tropical and 


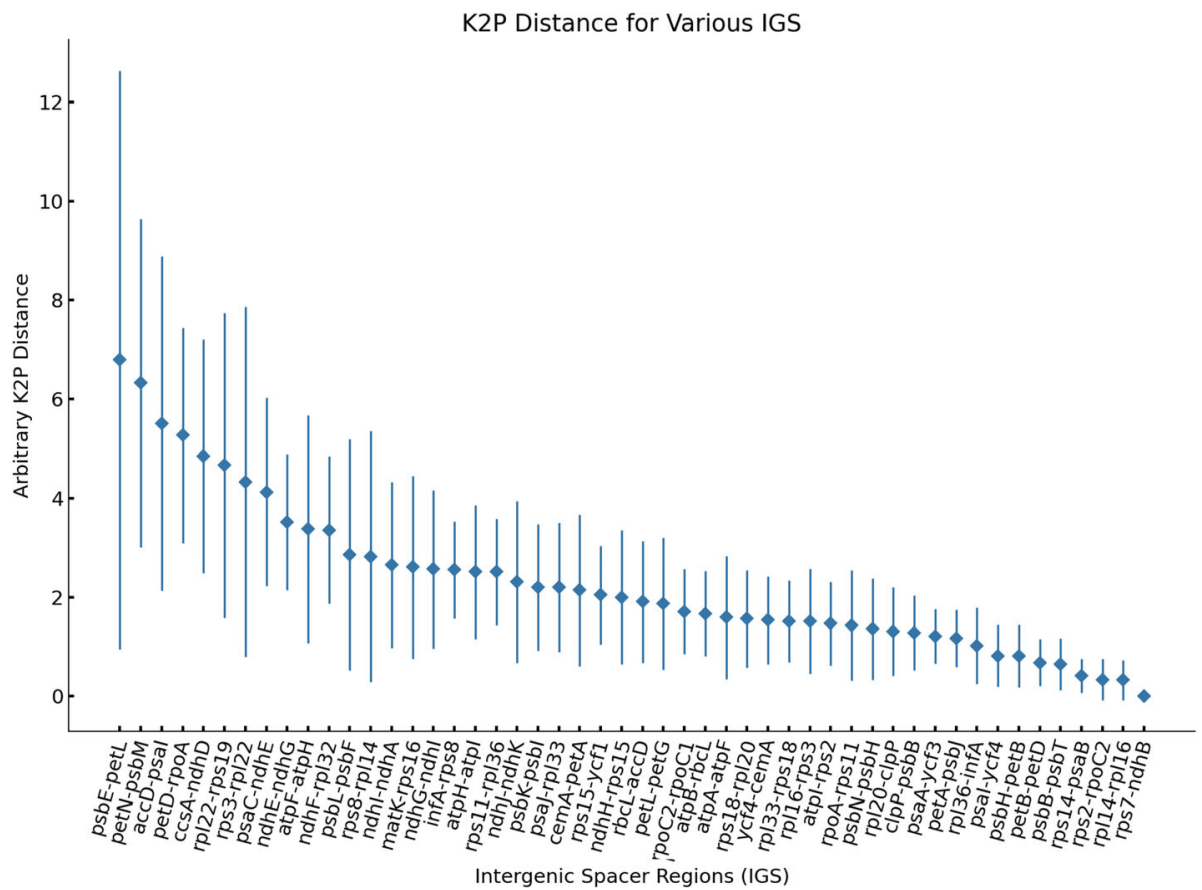

Fig. 4 Comparison of the variability of IGS regions among the plastomes of Alpinia galanga, A. hainanensis, A. nigra, A. officinarum, and A. oxyphylla. The $\mathrm{X}$-axis indicates the IGS regions, and the $\mathrm{Y}$-axis shows the range of $\mathrm{K} 2 \mathrm{p}$ distances between different pairs of species. The diamond shows the average K2p distance of the IGS region, respectively

subtropical areas. Their living environment's high temperature and humidity may be the reason for the positive selection of the $a c c \mathrm{D}$ and $y c f 1$ genes.

One of our goals is to develop markers that can distinguish the five medicinal Alpinia species. DNA markers derived from the plastomes have been widely used and are considered highly discriminatory for species identification such as Panax and Cruciata, including SNPs and InDels $[22,40]$. So far, these plastome-derived DNA markers are usually used to analyze intraspecies level diversity and phylogenetic analysis in Alpinia [20,21]. The most variable regions of the complete plastome can be used for DNA barcoding of closely related plant species [27]. Therefore, we developed the specific markers for discriminating Alpinia species based on the plastomes' hypervariable regions. The hypervariable regions identified in our study, such as $p e t \mathrm{~N}-p s b \mathrm{M}, p s a \mathrm{C}-n d h \mathrm{E}, a c c \mathrm{D}-$ $p s a \mathrm{I}$, were similar to those reported previously [19]. We found two markers derived from the pet $\mathrm{N}-p s b \mathrm{M}$ and psaJ-rpl33 IGS regions that successfully distinguished the five Alpinia species. The marker Alpp1 can't discriminate between A. officinarum and A. oxyphylla, because they are more closely related than with the other studied species. It has to be used combined with the marker Alpr1 for successful discrimination of the five Alpinia species.

Only a handful of Alpinia plastomes are sequenced and available in databases. Because the genus includes more than 200 spp., the information on the phylogeny of the genus is still rather limited. The complete Alpinia plastome sequences provided in this study expanded the taxonomic sampling and subsequently formulated new hypotheses about new potential relationships among Alpinia taxa [41]. From this point forward, additional plastomes of Alpinia species should be sequenced, which allow us to take a broad view of the evolutionary relationship and evolutionary processes of Alpinia species, lay the foundation for the further usage of these plants for the benefit of human lives. In this study, we developed molecular markers for the five Alpinia species that are of economic importance. With the identification of additional economically important Alpinia species, the same methodology can be used to identify their corresponding differentiating markers.

\section{Conclusions}

The complete plastomes of A. galanga, A. nigra, and A. officinarum are reported for the first time in this study. In addition, two molecular markers were developed from the hypervariable regions that can distinguish these five medicinal Alpinia species. The results obtained from these studies will contribute to our understanding of Alpinia classification, plastome evolution, and the discrimination of medicinal products derived from Alpinia species. 


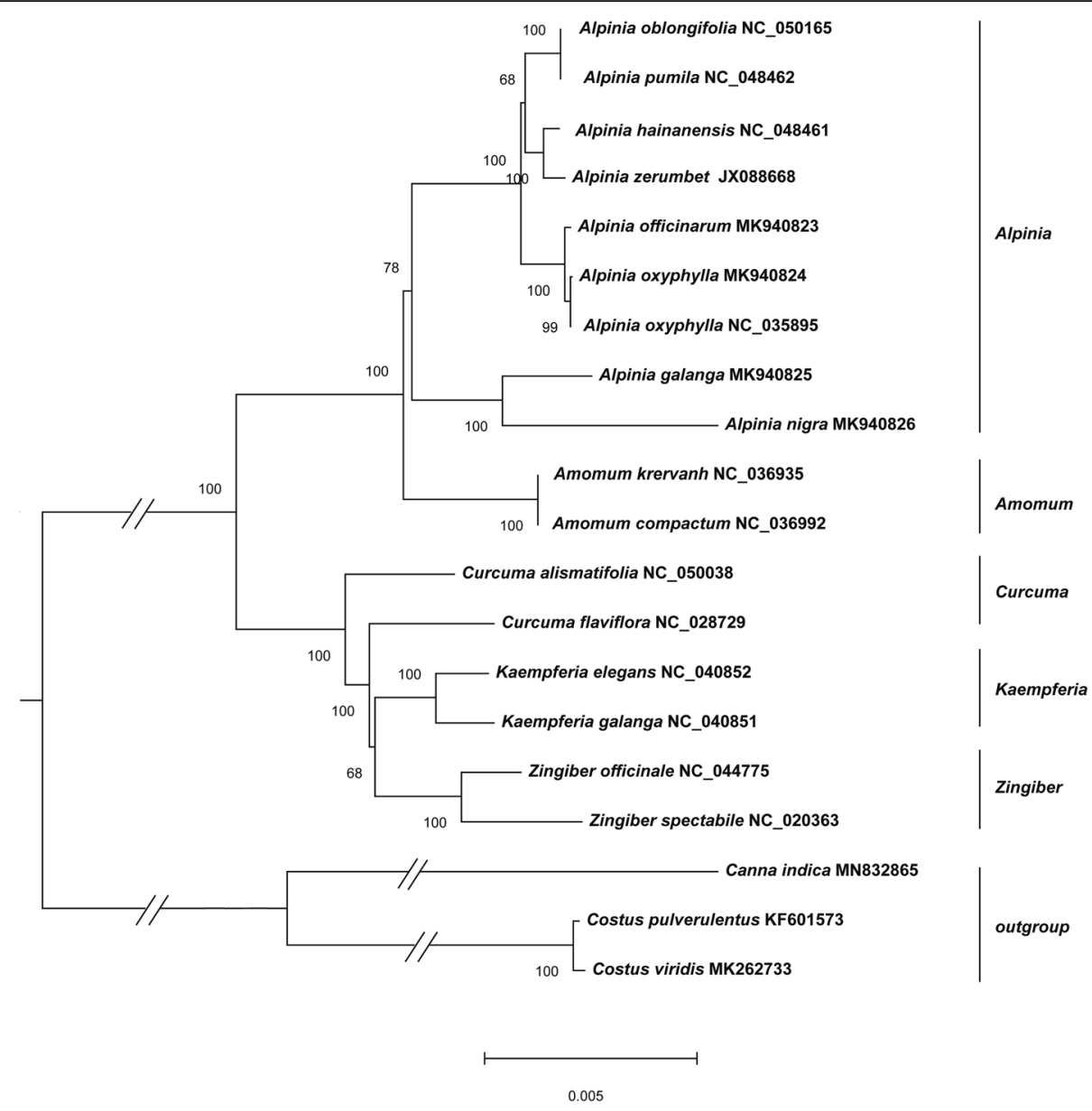

Fig. 5 Molecular phylogenetic tree based on common plastid genes. The phylogenetic tree was constructed with 77 shared genes present in 20 species using the maximum likelihood method implemented in Phylosuite. Costus pulverulentus, Costus viridis and Canna, were used as outgroups. Tribes to which each species belongs were shown to the right side of the tree. Bootstrap values were calculated with 1000 replicates

\section{Methods}

\section{Plant materials and total DNA preparation}

Fresh leaves were collected from plants grown in the Guangxi Medicinal Plant Garden in Nanning, Guangxi,
China (108 $\left.19^{\prime} \mathrm{E}, 22^{\circ} 51^{\prime} \mathrm{N}, 530,023\right)$, for the four species: A. galanga, A. nigra, A. officinarum, and A. oxyphylla. We collected these samples from five individual plants with different genotypes for each species for sequencing.

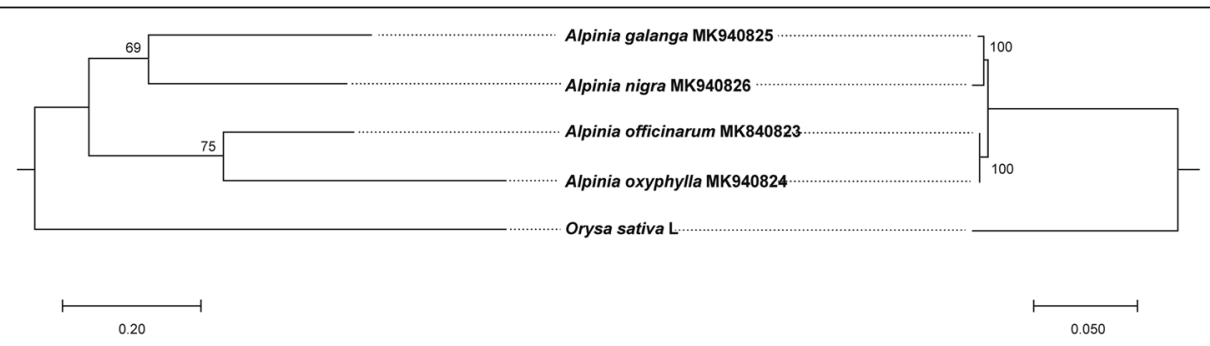

Fig. 6 Phylogenetic trees based on common genes identified using HybPiper pipeline and the shared DNA sequences of 77 protein-coding genes in the plastomes for the same five species. The phylogenetic tree on the left panel was constructed with the sequences of 4 shared contigs for nuclear genes present in 4 Alpinia species found by the HybPiper pipeline using the maximum likelihood method implemented in Phylosuite. The Oryza sativa L. was used as the outgroup. Bootstrap support scores were calculated from 1000 replicates. And the phylogenetic tree on the right panel was constructed with the shared DNA sequences of 77 protein-coding genes in the plastomes of the same five species in the nuclear tree using the same methods in phylogenomic analysis 
Table 4 Likelihood ratio tests to identify positively selected sites within the accD and ycf1 genes across 21 Alpinia plastomes

\begin{tabular}{|c|c|c|c|c|c|}
\hline Gene & Model compared & $\mathrm{Df}^{\mathrm{a}}$ & $-2 d / n L^{b}$ & $p$-values of $\mathrm{LRT}^{\mathrm{c}}$ & Positively selected sites $^{d}$ \\
\hline \multirow[t]{3}{*}{$a c c D$} & M1a (neutral) vs. M2a (selection) & 2 & 17.060598 & 0.000197396 & \\
\hline & M7 (beta) vs. M8 (beta $\& \omega>1$ ) & 2 & 17.143904 & 0.000189343 & $\begin{array}{l}1 \mathrm{~F} 0.995^{* *} \\
24 \mid 0.997^{* *} \\
181 \mathrm{~N} 0.977^{*}\end{array}$ \\
\hline & M8a $(\omega=1)$ vs. M8 (selection) & 1 & 17.051707 & 0.000036376 & \\
\hline \multirow[t]{3}{*}{ ycf1 } & M1a (neutral) vs. M2a (selection) & 2 & 17.751095 & 0.000139765 & \\
\hline & M7 (beta) vs. M8 (beta \& $\omega>1$ ) & 2 & 15.933493 & 0.000346805 & $\begin{array}{l}3 \text { F 0.964*, } \\
141 \text { F 0.969*, } \\
259 \text { E 0.968*, } \\
303 \text { Y 0.965*, } \\
326 \text { L 0.990** }\end{array}$ \\
\hline & M8a $(\omega=1)$ vs. M8 (selection) & 1 & 15.839639 & 0.000068943 & \\
\hline
\end{tabular}

${ }^{\mathrm{a}}$ Degree of freedom

${ }^{\mathrm{b}}$ Difference between the log likelihood values

¿LRT Likelihood Ratio Test

${ }^{d}$ Sites potentially under positive selection, indicated by the high Empirical Bayes values ('*': > 0.95; "**': $\left.>0.99\right)$

The samples were silica-dried and stored at the Herbarium of the Institute of Medicinal Plant Development (voucher numbers: Implad201910413, Implad201910414, Implad20180327, and Implad20180362). To develop molecular markers of Alpinia species, we collected fresh leaves of another group from Guangxi Medicinal Plant Garden in Nanning, Guangxi, China, and the ginger garden of South China Botanical Garden, China (113 $36^{\prime}$ E, $\left.23^{\circ} 18^{\prime} \mathrm{N}, 510,650\right)$. All samples were collected with permission from the Garden authorities. Detailed information is shown in Table S27. A plant genomic DNA kit (Tiangen Biotech, Beijing, Co., Ltd.) was used to extract total DNAs. The purity of total DNA was evaluated using electrophoresis on $1.0 \%$ agarose gels. And the concentration was measured using a Nanodrop spectrophotometer 2000 (Thermo Fisher Scientific Inc., Waltham, MA, USA). This study complies with relevant institutional, national, and international guidelines and legislation.

\section{Plastome sequencing, assembly, and annotation}

The sequencing libraries of total DNA from each species were prepared using the TruSeq DNA Sample Prep Kit (Illumina, Inc., San Diego, CA, USA) following the manufacturer's instructions. The total DNA was sheared into fragments at approximately $500 \mathrm{bp}$ long for pairedend library construction. The libraries were sequenced on an Illumina HiSeq 3000 platform (Illumina Inc., San Diego, CA, USA). After obtaining the paired-end reads $(2 \times 250 \mathrm{bp})$, we downloaded the plastid genomes from the GenBank database (https://www.ncbi.nlm.nih.gov/ genome/organelle/). These plastome sequences were used to search against Illumina paired-end reads using BLASTn with an E-value cutoff of 1e-5. The filtered reads were considered plastome-related and used for the downstream genome assembly. SPAdes (v. 3.10.1) [42] and CLC Genomics Workbench (v. 7, QIAGEN, Aarhus, Denmark) were used for de novo assembly. The dot plot of the contigs and reference genome were constructed and visualized for evaluating the assembly quality. The contigs were subjected to reassembly using the Seqman module of Lasergene (v. 11.0, Madison, Wisconsin). Only one contig was obtained for each of the Alpinia species.

We used the CpGAVAS2 web server [43] was used to annotate the four genomes. Cutoffs for the E-values of BLASTn and BLASTx were set to 1e-10. After the prefiltering step, the number of top hits for annotation included in the reference gene sets was set to 10. Manual

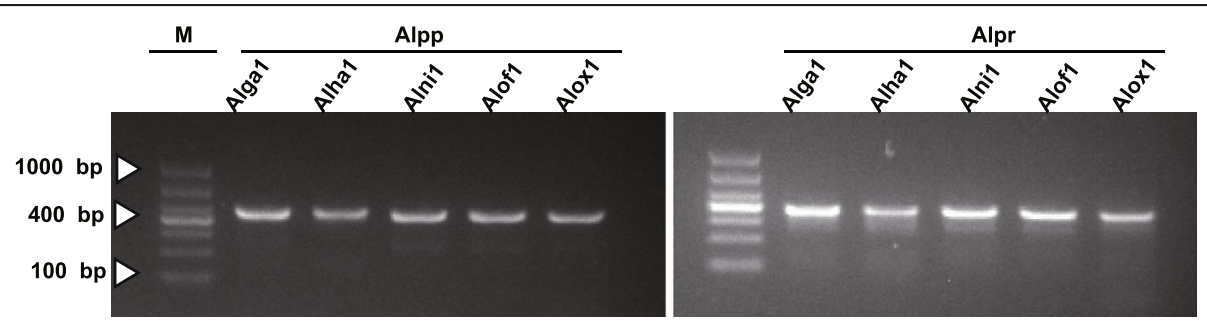

Fig. 7 The gel electrophoresis results of the amplification of DNA barcodes using designed primers. Lane M was the marker of DL1000. The lanes from left to right corresponded to products amplificated from the first individual of $A$. galanga, A. hainanensis, A. nigra, A. officinarum, and A. oxyphylla by primer Alpp and Alpr, respectively. The original uncropped image is shown in Figure S12 


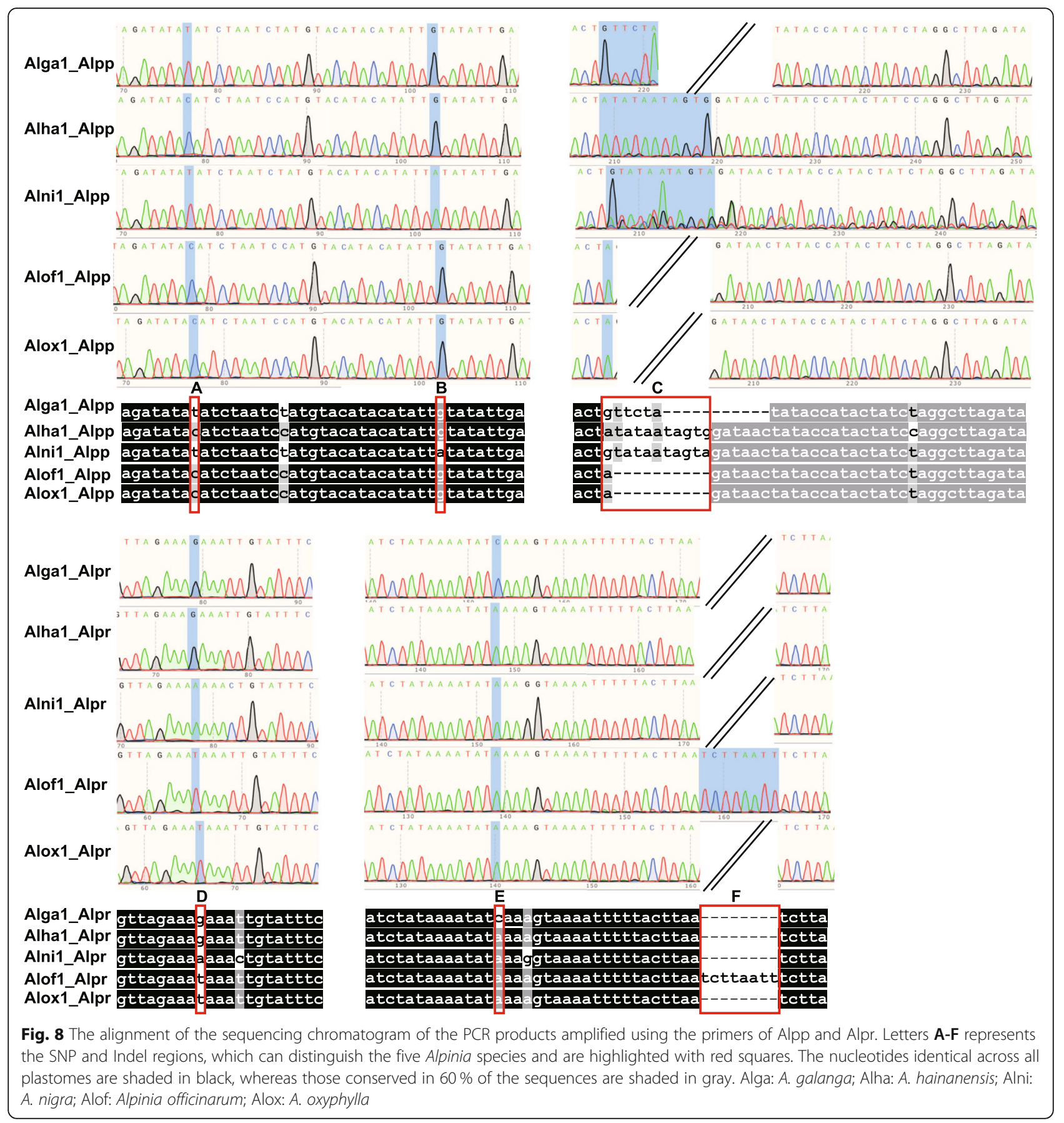

corrections were performed to determine the positions of the start and stop codons and the intron/exon boundaries. Codon usage frequency and GC content (i.e., the relative content of guanines and cytosines) were calculated using custom scripts. Their circular gene maps were drawn by the cpgview web server (http://www. herbalgenomics.org/cpgview/). The raw data and the annotated plastomes have been submitted to GenBank. The accession numbers of raw data were SRR9072115 (A. galanga), SRR9072120 (A. nigra), SRR9080445 (A. officinarum), and SRR9080447 (A. oxyphylla). The accession numbers of annotated plastomes were MK940825 (A. galanga), MK940826 (A. nigra), MK940823 (A. officinarum), and MK940824 (A. oxyphylla). We tested heteroplasmy patterns used NOVOPlasty in the four sequenced Alpinia species.

Repeat sequence analysis

SSRs were detected using the MISA Perl Script (http:// pgrc.ipk-gatersleben.de/misa/). The minimal numbers of 
repeat units are eight for mononucleotide repeats, four for di- and trinucleotide repeats, and three for tetra-, penta-, and hexanucleotide repeats. Long repeat sequences with a minimal length of $30 \mathrm{bp}$ and hamming distance $=3$ were predicted using REPuter [44]. Tandem repeat structures were scanned with Tandem Repeats Finder [45]. We set the parameters to 2 for matches and 7 for mismatches and Indels. In contrast, we set the minimum alignment score and maximum period size to 50 and 500, respectively. The minimum repeat size was $30 \mathrm{bp}$, and the cutoff for similarities among the repeat units was $90 \%$. All of the identified repeat structures were verified manually. Nested or redundant repeats were removed.

\section{Phylogenomic analysis}

For phylogenetic analyses, the DNA sequences of 77 protein-coding genes from 21 species of the Zingiberaceae family were extracted from the whole plastome sequences and aligned using MAFFT v.7 [46]. The 77 genes included $a c c \mathrm{D}, \operatorname{atp} \mathrm{A}, \operatorname{atp} \mathrm{B}, \operatorname{atp} \mathrm{E}, a t p \mathrm{~F}, a t p \mathrm{H}, a t p \mathrm{I}$, $\operatorname{ccs} \mathrm{A}, \operatorname{cem} \mathrm{A}, \operatorname{clp} \mathrm{P}, \inf \mathrm{A}, m a t \mathrm{~K}, n d h \mathrm{~A}, n d h \mathrm{~B}, n d h \mathrm{C}, n d h \mathrm{D}$, $n d h \mathrm{E}, n d h \mathrm{~F}, n d h \mathrm{G}, n d h \mathrm{H}, n d h \mathrm{I}, n d h \mathrm{~J}, n d h \mathrm{~K}, \operatorname{pet} \mathrm{A}, p e t \mathrm{~B}$, pet $\mathrm{D}, p e t \mathrm{G}, p e t \mathrm{~L}, p e t \mathrm{~N}, p s a \mathrm{~A}, p s a \mathrm{~B}, p s a \mathrm{C}, p s a \mathrm{I}, p s a \mathrm{~J}$, $p s b \mathrm{~A}, p s b \mathrm{C}, p s b \mathrm{D}, p s b \mathrm{E}, p s b \mathrm{~F}, p s b \mathrm{H}, p s b \mathrm{I}, p s b \mathrm{~J}, p s b \mathrm{~K}$, $p s b \mathrm{~L}, p s b \mathrm{M}, p s b \mathrm{~N}, p s b \mathrm{~T}, r b c \mathrm{~L}, r p l 14, r p l 16, r p l 2, r p l 20$, $r p l 22, r p l 23, r p l 32, r p l 33, r p l 36$, rpoA, rрoВ, rpoC1, rрoС2, rps11, rps12, rps14, rps15, rps16, rps18, rps19, rps2, rps3, rps4, rps7, rps8, ycf1, ycf2, ycf3, and ycf4. All aligned gene sequences were concatenated, and the bestfit evolutionary model (JTT + F + I + G4) was selected following the Bayesian information criterion (BIC) scores computed by ModelFinder [47]. The maximum likelihood (ML) tree was constructed by IQTREE v1.6.10 [48] with 1000 non-parametric bootstrap replications, and Costus pulverulentus, Costus viridis, and Canna indica as the outgroup taxa. Finally, the consensus tree was visualized using the MEGA X software [49].

Identification of nuclear markers for phylogenetic analysis To explore the phylogenetic relationship implied by single-copy nuclear markers, we used the HybPiper v1.2 [50] to identify nuclear markers among the Angiosperms-mega 353 gene set [28] from our sequencing reads for the four Alpinia species and then used them for phylogenetic analysis. The command line is “./reads_first.py -b mega353.fasta -r sample_001.fastq sample_002.fastq --prefix sample_result -bwa”. The HybPiper package contains an internal reference set of 353 genes. It can identify genes from high-throughput sequencing results that are homologous to these 353 genes and extract them for phylogenetic analysis. In particular, the expanded Angiosperms353 target file [28], which is a drop-in replacement for the original
Angiosperms353 file [51] in the HybPiper analyses, was used to capture loci in our sequence reads. We identified the potential genes for phylogenetic analysis as follows. Firstly, we used the retrieval script in HybPiper to identify contigs matching each probe (https://github.com/ mossmatters/HybPiper). This was done using the reads first.py script. Secondly, the common genes among the four species were selected. Finally, the contigs of these genes were used to create a phylogeny. Briefly, a phylogenetic tree was constructed with the contigs of these nuclear genes as above by IQTREE v1.6.10 [48] with 1000 non-parametric bootstrap replications, except the best-fit model is $\mathrm{HKY}+\mathrm{F}$ and the outgroup is Oryza sativa $\mathrm{L}$. The phylogenetic analyses based on these nuclear sequences were conducted for the sample taxa as those based on the shared coding sequences of 77 protein-coding genes in the plastomes.

\section{Selective pressure analysis}

The levels of selective pressure for a protein-coding gene are measured by the ratio of nonsynonymous to synonymous substitutions $(\omega)$ [15]. To detect the Alpinia plastid genes that were under positive selection, we extracted 77 protein-coding genes common to the $21 \mathrm{Zin}$ giberaceae plastomes, performed multiple sequence alignment using MAFFT, and constructed a maximum likelihood (ML) tree using IQTREE v1.6.10 [48]. Then we calculated the ratio of nonsynonymous $(\mathrm{dN})$, synonymous $(\mathrm{dS})$ and $\omega(\mathrm{dN} / \mathrm{dS})$ values were using CodeML in PAML Version 4.9 [52] with a One-ratio model (model $=0$, seqtype $=1$, NSsites $=0$ ). If the $\omega$ value is $>1$, the Bayes empirical Bayes (BEB) method implemented in the program EasyCodeML which is called site models (seqtype $=1$, model $=0$, NSsites $=0,1,2,3,7,8)$ [53] were used to identify positively selected sites.

\section{Identification of the hypervariable regions}

We conducted a comparative genome analysis for the complete Alpinia plastomes using the software mVISTA (http://genome.lbl.gov/vista/mvista/submit.shtml) in the Shuffle-LAGAN mode. The annotated $Z$. spectabile plastome (NC_020363) was used as the reference in the analysis. To identify the most divergent regions, we wrote a custom script to extract the start and end of the IGS regions from the GenBank files for the five plastomes, together with the plastome of $A$. hainanensis. A total of 59 IGSs shared by the five Alpinia plastomes were identified. The sequences were extracted and aligned using the ClustalW2 (v. 2.0.12) program with options "-type $=$ DNA -gapopen $=10$-gapext $=2$ " [54]. Pairwise distances were calculated using the K2p evolution model implemented in the distmat program from the EMBOSS package (v. 6.3.1) [55]. 


\section{Identification and validation of molecular markers for species discrimination}

We used variable intergenic regions to discriminate the five medicinal Alpinia species as a template to develop molecular markers. Primers were designed using the Primer3 program (http://bioinfo.ut.ee/primer3-0.4.0/). PCR amplifications were performed in a final volume of 25 $\mu \mathrm{L}$ with $12.5 \mu \mathrm{L} 2 \times$ Taq PCR Master Mix, $0.4 \mu \mathrm{M}$ of each primer, $2 \mu \mathrm{L}$ template DNA, and $10.1 \mu \mathrm{L}$ ddH2O. All amplifications were carried out in a Pro-Flex PCR system (Applied Biosystems, Waltham, MA, USA) under the following conditions: denaturation at $94{ }^{\circ} \mathrm{C}$ for 2 min, followed by 35 cycles of $94{ }^{\circ} \mathrm{C}$ for $30 \mathrm{~s}$, at specific annealing temperature (Tm) for $30 \mathrm{~s}, 72{ }^{\circ} \mathrm{C}$ for $60 \mathrm{~s}$ and $72{ }^{\circ} \mathrm{C}$ for $2 \mathrm{~min}$ as the final extension. PCR amplicons were visualized on $1.5 \%$ agarose gels and then subjected to Sanger sequencing on an ABI $3730 \times 1$ instrument (Applied Biosystems, USA) using the same set of primers used for PCR amplification.

\section{Abbreviations}

SSRs: Simple sequence repeats; IR: Inverted repeat; LSC: Long Single Copy; SSC: Short Single Copy; IGS: Intergenic spacer; $d N$ : The ratio of nonsynonymous; dS: The ratio of synonymous; $\omega$ : $d N / d S$; SNP: Single nucleotide polymorphism; Indel: Insertion-deletion mutations; ITS: Internal transcribed spacer; tRNA: Transfer RNA; rRNA: Ribosomal RNA

\section{Supplementary Information}

The online version contains supplementary material available at https://doi. org/10.1186/s12870-021-03204-1.

Additional file 1: Figure S1. Schematic representation of the A. nigra plastome features. Figure S2. Schematic representation of the $A$. officinarum plastome features. Figure S3. Schematic representation of the A. oxyphylla plastome features. Figure S4. The schematic diagram of position and length of introns and exons for the splitting genes in the plastome of A. galanga. The gene rps 12 was a trans-splicing gene. Figure S5. The schematic diagram of position and length of introns and exons for the splitting genes in the plastome of A. nigra. The gene rps 12 was a trans-splicing gene. Figure S6. The schematic diagram of position and length of introns and exons for the splitting genes in the plastome of $A$. officinarum. The gene rps12 was a trans-splicing gene. Figure S7. The schematic diagram of position and length of introns and exons for the splitting genes in the plastome of A. oxyphylla. The gene rps12 was a trans-splicing gene. Figure S8. The VCF output for the A. officinarum. Figure S9. The VCF output for the A. oxyphylla. Figure S10. The VCF output for the A. oxyphylla. Figure S11. The VCF output for the A. nigra. Figure S12. The original and full-length gel electrophoresis results of the amplification of DNA barcodes using designed primers. Figure S13. The alignment of amplicons produced by designed Alpp primers. Figure S14. The alignment of amplicons produced by designed Alpr primers. Figure S15. The alignment of amplicons in 10 Alpinia plastomes produced by designed Alpp primers in silico. Figure S16. The alignment of amplicons in 10 Alpinia plastomes produced by designed Alpp primers in silico.

Additional file 2: Table S1. Base composition in the plastomes of four Alpinia species. Table S2. List of genes annotated in the plastome of A galanga. Numbers in parentheses represented the repetition of genes. Superscript T: trans-splicing gene. Table S3. List of genes annotated in the plastome of A. nigra. Numbers in parentheses represented the repetition of genes. Superscript T: trans-splicing gene. Table S4. List of genes annotated in the plastome of A. officinarum. Numbers in parentheses represented the repetition of genes. Superscript T: trans-splicing gene.
Table S5. List of genes annotated in the plastome of A. oxyphylla. Numbers in parentheses represented the repetition of genes. Superscript T: trans-splicing gene. Table S6. The length of introns and exons for the splitting genes in the plastome of A. galanga. The gene rps12 was a trans-splicing gene. Table S7. The length of introns and exons for the splitting genes in the plastome of A. nigra. The gene rps12 was a transsplicing gene. Table S8. The length of introns and exons for the splitting genes in the plastome of A. officinarum. The gene rps12 was a transsplicing gene. Table S9. The length of introns and exons for the splitting genes in the plastome of A. oxyphylla. The gene rps12 was a transsplicing gene. Table S10. SSR identified in the plastome of A.galanga. P1 = Mononucleotide; P2 = Di nucleotide; P3 = Tri nucleotide; P4 = Tetra nucleotide; P5 = Penta nucleotide; $6=$ Hexa nucleotide repeats and $\mathrm{c}=$ Compound repeat microsatellites. Table S11. SSR identified in the plastome of A.nigra. P1 = Mononucleotide; P2 = Di nucleotide; P3 = Tri nucleotide; P4 = Tetra nucleotide; P5 = Penta nucleotide; $6=$ Hexa nucleotide repeats and $c=$ Compound repeat microsatellites. Table S12. SSR identified in the plastome of A. officinarum. P1 = Mononucleotide; P2 = Di nucleotide; P3 = Tri nucleotide; P4 = Tetra nucleotide; P5 = Penta nucleotide; $6=$ Hexa nucleotide repeats and $c=$ Compound repeat microsatellites. Table S13. SSR identified in the plastome of A. oxyphylla. P1 = Mononucleotide; P2 = Di nucleotide; P3 = Tri nucleotide; P4 = Tetra nucleotide; P5 = Penta nucleotide; $6=$ Hexa nucleotide repeats and $\mathrm{c}=$ Compound repeat microsatellites. Table S14. Comparison of SSR markers found among four Alpinia species and one outgroup species of Zingiber spectabile. Zisp: Zingiber spectabile; Alga: Alpinia galanga; Alni: Alpinia nigra; Alof: Alpinia officinarum; Alox: Alpinia oxyphylla. Table S15. Dispersed repeat sequences in the plastome of A. galanga. Table S16. Dispersed repeat sequences in the plastome of A.nigra. Table S17. Dispersed repeat sequences in the plastome of A. officinarum. Table S18. Dispersed repeat sequences in the plastome of A. oxyphylla. Table S19. Tandem repeat sequences identified in the plastome of A. galanga. Table S20. Tandem repeat sequences identified in the plastome of A. nigra. Table S21. Tandem repeat sequences identified in the plastome of A.officinarum. Table S22. Tandem repeat sequences identified in the plastome of A.oxyphylla. a: coding sequences; b: intergenic spacers. Table S23. The distances among the shared intergenic spacer (IGS) regions from the five Alpinia plastomes. Alga: Alpinia galanga; Alha: Alpinia hainanensis; Alni: Alpinia nigra; Alof: Alpinia officinarum; Alox: Alpinia oxyphylla. Table S24. The list of accession numbers of the plastome sequences used in the phylogenetic analyses of the Zingiberaceae. Table S25. The $d N, d S$ and $d N / d S(\omega)$ value of 77 commom protein-coding genes from plastomes of 21 Alpinia species. Table S26. The two pairs of primers for the ampilification of DNA barcodes. Table S27. The list of sample numbers of the samples used in the species discrimination analyses of the Alpinia.

\section{Acknowledgements}

The authors appreciate the taxonomists of the Guangxi Botanical Garden of Medicinal Plants who helped collect Alpinia samples from Guangxi. We also thank Ms. Xi Wu for supporting studies of phylogenetic analysis based on nuclear markers found by the HybPiper pipeline.

\section{Authors' contributions}

$\mathrm{CL}$ contributed to the conception and planning of the research. YHY performed the experiments and analyzed the data. LQW assembled the plastomes and performed data analysis. HMC and SYL performed the experiments. WWW and HMC collected samples. MJ extracted DNA for nextgeneration sequencing. $\mathrm{CL}, \mathrm{YHY}$, and LQW wrote the paper. JHW critically reviewed the manuscript. All authors have read and agreed on the final contents of the manuscript. The author(s) read and approved the final manuscript.

\section{Funding}

This work was supported by funds from The National Mega-Project for Innovative Drugs of China [2019ZX09735-002], National Natural Science Foundation of China [81872966], National Science \& Technology Fundamental Resources Investigation Program of China [2018FY100705], the CAMS Innovation Fund for Medical Sciences (2017-I2M-1-013, 2016-I2M-3-016) from the Chinese Academy of Medical Science. The funders were not involved in 
the study design, data collection, analysis, decision to publish, or manuscript preparation.

\section{Availability of data and materials}

The datasets generated during the current study are available in the GenBank: MK940823 and https://www.ncbi.nIm.nih.gov/nuccore/MK940823.1 for Alpinia officinarum, MK940824 and https://www.ncbi.nlm.nih.gov/nuccore/ MK940824.1 for A. oxyphylla, MK940825 and https://www.ncbi.nlm.nih.gov/ nuccore/MK940825.1 for A. galanga, MK940826 and https://www.ncbi.nlm. nih.gov/nuccore/MK940826.1 for A. nigra, respectively. Raw sequence data for this study also can be found in GenBank. The associated BioProject, SRA, and Bio-Sample numbers and the associated links are PRJNA543348, SRS4779543, SAMN11664063, and https://www.ncbi.nlm.nih.gov/ bioproject/543348 for Alpinia officinarum; PRJNA543352, SRS4779545, SAMN11664207, and https://www.ncbi.nlm.nih.gov/bioproject/543352 for A. oxyphylla; PRJNA543223, SRS4772960, SAMN11658378, and https://www.ncbi. nlm.nih.gov/bioproject/543223 for A. galanga; PRJNA543223, SRS4772960, SAMN11658378, and https://www.ncbi.nlm.nih.gov/bioproject/543239 for A. nigra, respectively.

\section{Declarations}

\section{Ethics approval and consent to participate}

All samples in this study were collected with permission from the Garden authorities.

This study complies with relevant institutional, national, and international guidelines and legislation.

\section{Consent for publication}

Not applicable.

\section{Competing interests}

The authors declare no competing interests.

\section{Author details}

${ }^{1}$ School of Environmental Science and Engineering, Tianjin University, 300072 Tianjin, China. ${ }^{2}$ Institute of Medicinal Plant Development, Chinese Academy of Medical Sciences and Peking Union Medical College, 100193 Beijing, People's Republic of China. ${ }^{3}$ College of Pharmacy, Heze University, Shandong Province 274015 Heze, People's Republic of China. ${ }^{4}$ Guangxi Botanical Garden of Medicinal Plants, 530023 Nanning, Guangxi, China. ${ }^{5}$ Department of Medical Data Sharing, Institute of Medical Information \& Library, Chinese Academy of Medical Sciences \& Peking Union Medical College, 100020 Beijing, China.

\section{Received: 10 April 2021 Accepted: 31 August 2021}

Published online: 22 September 2021

\section{References}

1. Kress WJ, Prince LM, Williams KJ. The phylogeny and a new classification of the gingers (Zingiberaceae): evidence from molecular data. Am J Bot. 2002; 89(10):1682-96.

2. $\quad$ Sirirugsa P. Thai Zingiberaceae: species diversity and their uses. Pure Appl Chem. 1999;70:1-8.

3. Larsen K, Lock J, Maas H, Maas P. Zingiberaceae. In: Kubitzki K, editor. Flowering plants. monocotyledons. The families and genera of vascular plants. Berlin, Heidelberg: Springer Netherlands; 1998. p. 474-95.

4. Kress WJ, Liu AZ, Newman M, Li QJ. The molecular phylogeny of Alpinia (Zingiberaceae): a complex and polyphyletic genus of gingers. Am J Bot. 2005;92(1):167-78.

5. Raut JS, Karuppayil SM. Bioprospecting of plant essential oils for medicinal uses. In: Fulekar M, Pathak B, Kale R, editors. Environment and sustainable development. New Delhi: Springer Netherlands; 2014. p. 59-76.

6. Khattak S, Saeed-ur-Rehman, Ullah Shah H, Ahmad W, Ahmad M. Biological effects of indigenous medicinal plants Curcuma longa and Alpinia galanga. Fitoterapia. 2005;76(2):254-257.

7. Matsuda H, Nakashima S, Oda Y, Nakamura S, Yoshikawa M. Melanogenesis inhibitors from the rhizomes of Alpinia officinarum in B16 melanoma cells. Bioorg Med Chem. 2009;17(16):6048-6053.
8. Guan S, Bao YM, Jiang B, An LJ. Protective effect of protocatechuic acid from Alpinia oxyphylla on hydrogen peroxide-induced oxidative PC12 cell death. Eur J Pharmacol. 2006;538(1-3):73-9.

9. Yang Y, Kinoshita K, Koyama K, Takahashi K, Tai T, Nunoura Y, Watanabe K. Two novel anti-emetic principles of Alpinia katsumadai. J Nat Prod. 1999; 62(12):1672-4.

10. Baruah D, Yadav RNS, Yadav A, Das AM. Alpinia nigra fruits mediated synthesis of silver nanoparticles and their antimicrobial and photocatalytic activities. J Photochem Photobiol B. 2019;201:111649.

11. Lin Q. Identification of Alpinia katsumadai and its mixed products of $A$. blepharocalyx and A. zerumbet. Strait Pharm J. 2006;18(4):102-3.

12. Zhao ZL, Zhou KY, Dong H, Xu LS. Characters of nrDNA ITS region sequences of fruits of Alpinia galanga and their adulterants. Planta Med. 2001;67(4):381-3.

13. Liu L, Wang Y, He P, Li P, Lee J, Soltis DE, Fu C. Chloroplast genome analyses and genomic resource development for epilithic sister genera Oresitrophe and Mukdenia (Saxifragaceae), using genome skimming data. BMC Genomics. 2018;19(1):235

14. Jiang $M$, Chen $H$, He S, Wang L, Chen AJ, Liu C. Sequencing, characterization, and comparative analyses of the plastome of Caragana rosea var. rosea. Int J Mol Sci. 2018;19(5):1419.

15. Song F, Li T, Burgess KS, Feng Y, Ge XJ. Complete plastome sequencing resolves taxonomic relationships among species of Calligonum L. (Polygonaceae) in China. BMC Plant Biol. 2020;20(1):261.

16. Greiner S, Wang X, Rauwolf U, Silber MV, Mayer K, Meurer J, Haberer G, Herrmann RG. The complete nucleotide sequences of the five genetically distinct plastid genomes of Oenothera, subsection Oenothera: I. sequence evaluation and plastome evolution. Nucleic Acids Res. 2008;36(7):2366-2378.

17. Niu Z, Xue Q, Zhu S, Sun J, Liu W, Ding X. The complete plastome sequences of four Orchid species: insights into the evolution of the Orchidaceae and the utility of plastomic mutational hotspots. Front Plant Sci. 2017:8:715.

18. Gao B, Yuan L, Tang T, Hou J, Pan K, Wei N. The complete chloroplast genome sequence of Alpinia oxyphylla Miq. and comparison analysis within the Zingiberaceae family. PLoS One. 2019;14(6):e0218817.

19. Li DM, Zhu GF, Xu YC, Ye YJ, Liu JM. Plants (Basel). 2020;9(2):286.

20. Tan WH, Chai LC, Chin CF. Efficacy of DNA barcode internal transcribed spacer 2 (ITS 2) in phylogenetic study of Alpinia species from Peninsular Malaysia. Physiol Mol Biol Plants. 2020;26(9):1889-1896.

21. Basak S, Chakrabartty I, Hedaoo V, Shelke RG, Rangan L. Assessment of genetic variation among wild Alpinia nigra (Zingiberaceae) population: an approach based on molecular phylogeny. Mol Biol Rep. 2019;46(1):177-189.

22. Nguyen VB, Linh Giang VN, Waminal NE, Park HS, Kim NH, Jang W, Lee J, Yang TJ. Comprehensive comparative analysis of chloroplast genomes from seven Panax species and development of an authentication system based on species-unique single nucleotide polymorphism markers. J Ginseng Res. 2020;44(1):135-144.

23. Kim K, Lee SC, Lee J, Lee HO, Joh HJ, Kim NH, Park HS, Yang TJ. Comprehensive Survey of Genetic Diversity in Chloroplast Genomes and $45 \mathrm{~S}$ nrDNAs within Panax ginseng Species. PLoS One. 2015;10(6):e0117159.

24. Weitemier K, Straub SC, Fishbein M, Liston A. Intragenomic polymorphisms among high-copy loci: a genus-wide study of nuclear ribosomal DNA in Asclepias (Apocynaceae). PeerJ. 2015;3:e718.

25. Sullivan AR, Schiffthaler B, Thompson SL, Street NR, Wang XR. Interspecific plastome recombination reflects ancient reticulate evolution in Picea (Pinaceae). Mol Biol Evol. 2017:34(7):1689-701.

26. Zhu A, Guo W, Gupta S, Fan W, Mower JP. Evolutionary dynamics of the plastid inverted repeat: the effects of expansion, contraction, and loss on substitution rates. New Phytol. 2016;209(4):1747-56.

27. Dong W, Liu J, Yu J, Wang L, Zhou S. Highly variable chloroplast markers for evaluating plant phylogeny at low taxonomic levels and for DNA barcoding. PLoS One. 2012;7(4):e35071.

28. McLay TG, Birch JL, Gunn BF, Ning W, Tate JA, Nauheimer L, Joyce EM, Simpson L, Simpson L, Schmidt-Lebuhn AN, Baker WJ, Forest F, Jackson, CJ. New targets acquired: improving locus recovery from the Angiosperms353 probe set. Applications in Plant Sciences. 2021;9(7):e11420.

29. Ni L, Zhao Z, Xu H, Chen S, Dorje G. The complete chloroplast genome of Gentiana straminea (Gentianaceae), an endemic species to the SinoHimalayan subregion. Gene. 2016;577(2):281-288.

30. Kress W, Newman M, Poulsen A, Specht C. An analysis of generic circumscriptions in tribe Alpinieae (Alpinioideae: Zingiberaceae). Gardens Bull Singapore. 2007;59:113-28. 
31. Selvaraj D, Sarma RK, Sathishkumar R. Phylogenetic analysis of chloroplast matK gene from Zingiberaceae for plant DNA barcoding. Bioinformation. 2008;3(1):24-7.

32. Theerakulpisut $P$, Triboun P, Mahakham W, Maensiri D, Khampila J, Chantaranothai P. Phylogeny of the genus Zingiber (Zingiberaceae) based on nuclear ITS sequence data. Kew Bulletin. 2012:67(3):389-395.

33. Ngamriabsakul C, Newman MF, Cronk QCB. The phylogeny of tribe Zingibereae (Zingiberaceae) based on ITS (nrDNA) and trnL-F (cpDNA) sequences. Edinburgh J Bot. 2004;60(3):483-507.

34. Erixon P, Oxelman B. Whole-Gene Positive Selection, Elevated Synonymous Substitution Rates, Duplication, and Indel Evolution of the Chloroplast CIPP1 Gene. Plos One. 2008;3(1):e1386.

35. Kapralov MV, Filatov DA. Widespread positive selection in the photosynthetic Rubisco enzyme. BMC Evol Biol. 2007;7(1):1-10.

36. Da CH, Shi LC, Pei GX. Molecular evolution and positive Darwinian selection of the chloroplast maturase matK. J Plant Res. 2010;123(2):241-7.

37. Li DM, Ye YJ, Xu YC, Liu JM, Zhu GF. Complete chloroplast genomes of Zingiber montanum and Zingiber zerumbet: Genome structure, comparative and phylogenetic analyses. PLoS One. 2020;15(7):e0236590.

38. Kode V, Mudd EA, lamtham S, Day A. The tobacco plastid accD gene is essential and is required for leaf development. Plant J. 2005;44(2):237-44.

39. de Vries J, Sousa FL, Bolter B, Soll J, Gould SB. YCF1: a green TIC? Plant Cell. 2015;27(7):1827-1833.

40. Zhou T, Wang J, Jia Y, Li W, Xu F, Wang X. Comparative chloroplast genome analyses of species in Gentiana section Cruciata (Gentianaceae) and the development of authentication markers. Int J Mol Sci. 2018;19(7):1962.

41. Nabhan AR, Sarkar IN. The impact of taxon sampling on phylogenetic inference: a review of two decades of controversy. Brief Bioinform. 2012; 13(1):122-34.

42. Bankevich A, Nurk S, Antipov D, Gurevich A, Dvorkin M, Kulikov A, Lesin V, Nikolenko S, Pham S, Prjibelski A, et al. SPAdes: a new genome assembly algorithm and its applications to single-cell sequencing. J Comput Biol. 2012;19(5):455-77.

43. Shi $L$, Chen $H$, Jiang M, Wang L, Wu X, Huang L, Liu C. CPGAVAS2, an integrated plastome sequence annotator and analyzer. Nucleic Acids Res. 2019;47(W1):W65-73.

44. Kurtz S, Choudhuri JV, Ohlebusch E, Schleiermacher C, Stoye J, Giegerich R REPuter: the manifold applications of repeat analysis on a genomic scale. Nucleic Acids Res. 2001;29(22):4633-42.

45. Benson G. Tandem repeats finder: a program to analyze DNA sequences. Nucleic Acids Res. 1999;27(2):573-80.

46. Katoh K, Standley DM. MAFFT multiple sequence alignment software version 7: improvements in performance and usability. Mol Biol Evol. 2013; 30(4):772-780

47. Kalyaanamoorthy S, Minh BQ, Wong TKF, von Haeseler A, Jermiin LS ModelFinder: fast model selection for accurate phylogenetic estimates. Nat Methods. 2017;14(6):587-589.

48. Nguyen LT, Schmidt HA, von Haeseler A, Minh BQ. IQ-TREE: a fast and effective stochastic algorithm for estimating maximum-likelihood phylogenies. Mol Biol Evol. 2015;32(1):268-274

49. Kumar S, Stecher G, Li M, Knyaz C, Tamura K. MEGA X: molecular evolutionary genetics analysis across computing platforms. Mol Biol Evol. 2018;35(6):1547-1549.

50. Johnson MG, Gardner EM, Liu Y, Medina R, Goffinet B, Shaw AJ, Zerega NJC, Wickett NJ. HybPiper: extracting coding sequence and introns for phylogenetics from high-throughput sequencing reads using target enrichment. Appl Plant Sci. 2016;4(7):1600016.

51. Johnson MG, Pokorny L, Dodsworth S, Botigue LR, Cowan RS, Devault A, Eiserhardt WL, Epitawalage N, Forest F, Kim JT. A universal probe set for targeted sequencing of 353 nuclear genes from any flowering plant designed using k-medoids clustering. Syst Biol. 2019;68(4):594-606.

52. Yang Z, Nielsen R. Codon-substitution models for detecting molecular adaptation at individual sites along specific lineages. Mol Biol Evol. 2002; 19(6):908-17.

53. Gao F, Chen C, Arab DA, Du Z, He Y, Ho SYW. EasyCodeML: A visual tool for analysis of selection using CodeML. Ecol Evol. 2019(7):3891-8.

54. Thompson JD, Gibson TJ, Higgins DG. Multiple sequence alignment using ClustalW and ClustalX. Curr Prot Bioinform. 2003:2.3.1-2.3.22.

55. Rice $P$, Longden I, Bleasby A. EMBOSS: the european molecular biology open software suite. Trends Genetics. 2000;16(6):276-7.

\section{Publisher's Note}

Springer Nature remains neutral with regard to jurisdictional claims in published maps and institutional affiliations.

\section{Ready to submit your research? Choose BMC and benefit from:}

- fast, convenient online submission

- thorough peer review by experienced researchers in your field

- rapid publication on acceptance

- support for research data, including large and complex data types

- gold Open Access which fosters wider collaboration and increased citations

- maximum visibility for your research: over $100 \mathrm{M}$ website views per year

At BMC, research is always in progress.

Learn more biomedcentral.com/submissions 